Original Research

\title{
Significant variations in surgical construct and return to sport protocols with syndesmotic injuries: an ISAKOS global perspective
}

\author{
Kenneth J. Hunt, MD ${ }^{\mathrm{a}, *}$, Jonathan Bartolomei, MS ${ }^{\mathrm{a}}$, Shanthan C. Challa, MD ${ }^{\mathrm{a}}$, \\ Jeremy J. McCormick, $\mathrm{MD}^{\mathrm{b}}$, Pieter D'Hooghe, $\mathrm{MD}, \mathrm{PhD}^{\mathrm{c}}$, Michael Tuffiash, MS ${ }^{\mathrm{a}}$, \\ Annunziato Amendola, MD ${ }^{\mathrm{d}}$, ISAKOS Leg, Ankle Foot Committee \\ ${ }^{a}$ University of Colorado - Denver, Department of Orthopedics, Denver, CO, USA \\ ${ }^{\mathrm{b}}$ Washington University School of Medicine, Department of Orthopedics, St. Louis, MO, USA \\ ${ }^{c}$ Aspetar Orthopaedic and Sports Medicine Hospital, Department of Orthopaedic Surgery, Doha, Qatar \\ d Duke University School of Medicine, Department of Orthopedic Surgery, Durham, NC, USA
}

\section{A R T I C L E I N F O}

\section{Keywords:}

Syndesmotic injury

Football

Sports injuries

Return to sport

High ankle sprain

\begin{abstract}
A B S T R A C T
Objectives: Although the body of literature on syndesmosis injuries is growing with regard to both the biomechanics and clinical outcomes for various fixation constructs, there is little consensus on the optimal treatment and return to sport strategy for these injuries. We endeavoured to assess the current approaches to managing syndesmotic injuries through a Research Electronic Data Capture survey.

Methods: The survey consisted of 27 questions, including respondent demographics, indications for treatment of syndesmotic injuries, preferred treatment and technique, and postoperative management. Responses were generated through six different athlete scenarios: moderate impact, high impact, and very high impact athletes with/without complete deltoid injury. Frequencies and percentages were calculated for all categorical responses. Results: A total of 742 providers responded to the survey, including 457 American surgeons and 285 members of various international societies. Flexible devices were the preferred fixation construct (47.1\%), followed by screws (29.6\%), hybrid fixation (e.g. combination of flexible device and screw, 18\%), and other (5.3\%). Sixty-four percent of respondents noted that their rehabilitation protocols would not change for each athlete scenario. Considerable variability was present in anticipated return to full participation, largely dependent on the presence or absence of a deltoid ligament injury.

Conclusion: The most common elements used as surgical indications were syndesmosis widening $>2 \mathrm{~mm}$ on $\mathrm{x}$-ray, an anterior inferior talofibular ligament injury in combination with a posterior inferior talofinular ligament or deltoid ligament involvement on magnetic resonance imaging, and widening of the distal tibiofibular joint during arthroscopic evaluation. Overall, flexible fixation (e.g. suture button) was the preferred device choice for the repair of an injured syndesmosis. Most respondents did not alter their rehab protocol or anticipated return to play timeline based on the injury severity. However, there was considerable variability between respondents on the time to weight-bearing, running, and full participation. Further pragmatic outcomes data are necessary to guide safe return to play protocols for syndesmotic injuries.

Level of evidence: Level IV.
\end{abstract}

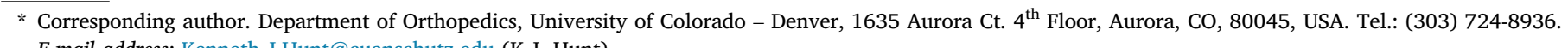
E-mail address: Kenneth.J.Hunt@cuanschutz.edu (K.J. Hunt). 


\section{What are the new findings?}

- The use of flexible devices is the most popular method of fixation for syndesmotic injuries, irrespective of the athlete scenario or the presence of a deltoid injury.

- A majority of responding surgeons (340/566, 63.7\%) indicated that they would use the same postoperative protocols for all athlete types and injury patterns presented in this survey.

- The results of the survey suggest that although there was consistency among responses at the individual level, there was considerable interrespondent variability with regard to the return to activity times for athletes with syndesmotic injuries.

\section{Introduction}

Ankle injuries are among the most common injuries sustained by athletes, accounting for approximately $10-30 \%$ of single-sport injuries [1]. Current literature suggests that syndesmotic ankle injuries constitute between $1 \%$ and $25 \%$ of all ankle sprains [1-4]. In comparison to other ankle sprains, injuries to the syndesmosis result in a higher degree of impairment, a longer recovery time, and an extended return to play for athletes $[1,3,4]$. Despite research and development of multiple techniques for syndesmotic fixation, there remains a lack of consensus regarding the best treatment option [5,7]. Until recently, open reduction and screw fixation were considered the standard treatment for syndesmotic injuries [4,6,8-10]. Flexible fixation using a suture button device (i.e. Arthrex Tightrope) has also become an increasingly common surgical treatment used for these injuries $[11,12]$.

Although many factors contribute to the range of outcomes following syndesmotic fixation in athletes, more detailed guidelines regarding operative indications, technique and postoperative management are of critical value to the physician caring for these athletes [13]. Mak et al. suggest that aggressive rehabilitation is a way to ensure the quickest return to sport [14]. However, we are not aware of any established postoperative rehabilitation protocols that have demonstrably decreased time to return to sport. To facilitate the development of a surgical and postoperative protocol that optimises return to competition, it is critical to understand current practices, as it relates to managing these injuries. A better understanding of current practices for the treatment of syndesmotic injuries, including techniques and postoperative management, will help to create a consensus guideline for syndesmotic injuries. In addition, issues that lack consensus or add controversy may stimulate further research in these areas.

The objective of this study was to determine current practices, as it relates to surgical indications, techniques, and return to sport for athletes with ligamentous syndesmotic injuries. We proposed hypothetical scenarios of injured athletes to determine indications for operation, device choice, and postoperative return to sport expectations. We hypothesised that (1) there will be variability in the management of syndesmotic injuries amongst all providers, (2) flexible fixation devices will be the preferred treatment method over rigid or hybrid fixation, and (3) return to activity times will change based on the type of athlete and the severity of their injury.

\section{Methods}

\section{Survey dissemination}

We recruited survey participants through six national and international orthopedic sports medicine and foot and ankle surgery societies after obtaining approval through our institutional review board. These societies included the International Society of Arthroscopy, Knee Surgery, and Orthopedic Sports Medicine, American Orthopedic Foot and Ankle Society, American Orthopedic Society for Sports Medicine, Belgian
Society of Orthopaedics and Traumatology, Canadian Orthopedic Foot and Ankle Society, and British Orthopedic Foot and Ankle Society. Invitations were distributed via email to the entire society membership, three times over a 6-week interval through the web-based Research Electronic Data Capture system.

\section{Survey components (Supplemental 1 and 2)}

Respondents answered demographical questions about their practice location and setting, society membership, and training background. Participants then responded to questions focused on their indications for the treatment of syndesmotic injuries and the data used during their decision-making process. We constructed six athlete scenarios with varying degrees of injury: moderate impact (e.g. a 180-pound tennis player), high impact (e.g. a 220-pound American football wide receiver), or very high impact athletes (e.g. a 300-pound American offensive or defensive lineman) either with or without a complete deltoid tear. The example of the athlete (tennis vs. American football player) in each scenario was used to provide surgeons with an athlete size and skillset to base their decision. Not operating on American football players was not an exclusion criterion for the study.

Each of the respondent's preferred technique, suture buttons, syndesmotic screws, or a combination of the two, was also queried for operative repair of indicated syndesmotic injury. Finally, the survey collected data on the surgeon's preferred duration of rehabilitation/ graduation to activity (weight-bearing, running, and full participation). The time for rehabilitation ranged from immediately after surgery to 6 months postoperatively, with an option to select "It depends" and enter a response.

\section{Statistical analysis}

We calculated the response frequency counts and percentages for all nominal and ordinal variables in IBM SPSS Statistics for Windows, version 26 (IBM Corp., Armonk, N.Y., USA). Survey data were filtered for missing values on an item-by-item basis, except for the primary items in the survey sections related to preferred fixation device and postoperative

Table 1

Surgeon background by location, practice setting, subspecialty, and fellowship training.

\begin{tabular}{|c|c|c|}
\hline & North America & International \\
\hline Location & n (\%) & n (\%) \\
\hline Continent/country & $\begin{array}{l}\text { United States }=407 \\
\text { Canada }=47 \\
\text { Other }=3\end{array}$ & $\begin{array}{l}\text { Europe }=172 \\
\text { Asia }=71 \\
\text { Central } \text { or } S . \text { America }=30 \\
\text { Australia and Oceania }=10 \\
\text { Africa }=1\end{array}$ \\
\hline Practice setting & n (\%) & n (\%) \\
\hline Private practice & $247(54.0)$ & $85(30.8)$ \\
\hline Academic medical centre & $127(27.8)$ & $137(49.6)$ \\
\hline Non-academic hospital & $68(14.9)$ & $49(17.8)$ \\
\hline Rural & $15(3.3)$ & $5(1.8)$ \\
\hline Practice subspecialty $^{\mathrm{a}}$ & n (\%) & n (\%) \\
\hline Foot and ankle & $321(70.2)$ & $136(49.3)$ \\
\hline Sports medicine & $93(20.3)$ & $94(40.0)$ \\
\hline Trauma/fracture care & $38(8.3)$ & $35(12.6)$ \\
\hline Surgical fellowship & n (\%) & n (\%) \\
\hline Foot and ankle & $344(66.5)$ & $159(40.9)$ \\
\hline Sports medicine & $91(17.6)$ & $125(32.1)$ \\
\hline Orthopaedic trauma & $36(7.0)$ & $63(16.2)$ \\
\hline Other & $16(3.1)$ & $16(4.1)$ \\
\hline None & $30(5.8)$ & $26(6.7)$ \\
\hline
\end{tabular}

a Proportion of surgeons who identified a subspecialty as $50 \%$ or more of their patient population (total proportions may not equal 100\% due to surgeons practicing in multiple subspecialties). 
return to play protocols. We only reported responses to these latter two survey sections for those surgeons who provided valid responses to all six athletes/injury scenarios ( $n=566$ for preferred device items; $n=533$ for postoperative return to activity items).

\section{Results}

\section{Demographics}

We collected 742 total responses, with 543 respondents $(73.1 \%)$ completing all of the required items and 199 (26.9\%) responding to a portion of the survey. A majority of respondents (62\%) reported their surgical practices in North America (457), followed by Europe (172, $23 \%$ ), and then Asia (71, 10\%; Table 1). A plurality (45.1\%) of the respondents practiced in a private practice setting (333), followed by academic medical centres (264, 35.7\%), non-academic hospitals (121, $16.4 \%)$, and rural settings (21, 2.8\%; Table 1$)$.

\section{Indications for operative treatment}

Magnetic resonance imaging, radiographs, and arthroscopy

Most respondents (63.9\%) specified that magnetic resonance imaging (MRI) findings alone are not sufficient as a sole indication for surgery. Less than a quarter $(145 / 609 ; 23.8 \%)$ of respondents indicated that MRI evidence of an interosseous membrane (IOM) injury $>5 \mathrm{~cm}$ above the syndesmosis would be a sole indication for operative treatment. MRI evidence of a posterior inferior talofibular ligament (PITFL) or deltoid injury accounted for $20.7 \%$ of sole indications for operative treatment responses $(63 / 612 ; 10.2 \%$ and 64/609; 10.5\%, respectively; Table 2). Seventy-one percent of survey participants (437/611) reported radiographic evidence of $>2 \mathrm{~mm}$ of medial clear space widening on stress radiography as a sole indication for operative treatment. Finally, almost three-fourths of respondents $(461 / 617,74.7 \%)$ used $>2 \mathrm{~mm}$ of syndesmosis widening as their sole indication for surgery (Table 2). Arthroscopic finding of syndesmotic widening was used as the sole indication

Table 2

Proportions of surgeons endorsing common diagnostic criteria as sole or contributing indications to repair a syndesmotic ankle injury (SAI).

\begin{tabular}{|c|c|c|}
\hline & $\begin{array}{l}\text { Sole } \\
\text { indication }\end{array}$ & $\begin{array}{l}\text { Contributing } \\
\text { indication }\end{array}$ \\
\hline Radiology & n (\%) & n (\%) \\
\hline Syndesmosis widening $\geq 2 \mathrm{~cm}$ & $461(74.7)$ & $156(25.3)$ \\
\hline Medial clear space widening $\geq 2 \mathrm{~cm}$ & 437 (71.5) & $174(28.5)$ \\
\hline MRI & n (\%) & n (\%) \\
\hline IOM injury $>5 \mathrm{~cm}$ above syndesmosis & $145(23.8)$ & $464(76.2)$ \\
\hline Deltoid ligament injury & $64(10.5)$ & 545 (89.5) \\
\hline PITFL injury & $63(10.3)$ & 549 (89.7) \\
\hline Physical examination & n (\%) & n (\%) \\
\hline Positive lateral translation (Cotton) test & $244(40.1)$ & 365 (59.9) \\
\hline Positive fibular translation test & $230(37.9)$ & $377(62.1)$ \\
\hline Tenderness with external rotation & $45(7.4)$ & $566(92.6)$ \\
\hline $\begin{array}{l}\text { Tenderness at IOM }>5 \mathrm{~cm} \text { above } \\
\text { syndesmosis }\end{array}$ & $38(6.2)$ & $573(93.8)$ \\
\hline Single/standing limb heel rise test & $31(5.1)$ & 575 (94.9) \\
\hline Tenderness at AITFL & $25(4.1)$ & $591(95.9)$ \\
\hline Tenderness at PITFL & $16(2.6)$ & $597(97.4)$ \\
\hline Arthroscopic procedure & n (\%) & n (\%) \\
\hline Syndesmosis widening $^{\mathrm{a}}$ & $410(68.6)$ & 165 (27.6) \\
\hline Medial clear space widening ${ }^{a}$ & $284(47.5)$ & $286(47.8)$ \\
\hline
\end{tabular}

AITFL = anterior inferior talofibular ligament; IOM = interosseous membrane; MRI = magnetic resonance imaging; PITFL $=$ posterior inferior talofibular ligament.

a Additional 4\% of surgeons responded that they would not use either arthroscopic finding as an indication to repair syndesmotic ankle injury (SAI). for surgical repair for over two-thirds of participants (410/598, 68.5\%). The remaining responses included arthroscopic findings of syndesmotic widening in combination with other findings as a sole (165/598, 27.6\%) or rare $(23 / 598,3.8 \%)$ indication for operative fixation. Medial clear space widening on arthroscopy exhibited no consensus in its use for operative decision-making, as $47.5 \%$ (284/598), $47.8 \%$ (286/598), and $4.7 \%(28 / 598)$ reported this finding as a sole, in combination, or rare indication for surgery, respectively (Table 2).

\section{Physical examination}

A positive Cotton test was the most commonly selected physical examination finding that would indicate a need for surgery $(244 / 609$, $40 \%)$. Other physical examination findings used as sole indications included pain with external rotation (45/609, 7.4\%), tenderness around the IOM $>5 \mathrm{~cm}$ superior to the syndesmosis $(38 / 609,6.2 \%)$, inability to perform a single-leg heel-rise $(31 / 609,5.1 \%)$, and tenderness along the anterior $(25 / 609 ; 4.1 \%)$ and posterior $(16 / 609 ; 2.6 \%)$ syndesmosis ligaments (Table 2).

\section{Deltoid injury and foot alignment}

Most respondents indicated that they would "never or almost never" repair the deltoid or address this injury "less than half" of the time (191/ $565,33.8 \%$ and $157 / 565,27.7 \%$, respectively). Foot alignment had little impact as only $13.7 \%(73 / 459)$ of respondents reported this clinical finding as a reason to alter their treatment of a syndesmosis injury. Pes planovalgus feet had the greatest effect in this cohort, with 78.1\% (57/ 73) noting this alignment as a reason to change their management. Conversely, pes cavus alignment only altered the treatment decision of $38.7 \%(29 / 73)$ of surgeons.

\section{Athlete scenarios}

\section{Moderate impact athlete}

For this athlete population (e.g. a 180-lb tennis player), one or two flexible devices were the most popular choice $(23 \%$ and $40 \%$, respectively). The presence of a deltoid injury only changed the response of $22.6 \%(169 / 566)$ of respondents, with two flexible devices and two screws across four cortices being the most selected options (211/566, $37.3 \%$ and $80 / 566,14.1 \%$, respectively). The most commonly selected postoperative management protocols for the moderate impact athlete after surgery were 6 weeks for weight-bearing and 12 weeks until running and full participation. Athletes who had an additional deltoid injury were most frequently held from full participation for 16 weeks after surgery in this hypothetical scenario (Fig. 1).

\section{High impact athlete}

High impact athletes (e.g. 220-lb American football wide receivers) are most commonly treated with two flexible devices $(255 / 566$, $45.1 \%)$. More than one-quarter of respondents (27.2\%) noted that the presence of a deltoid injury would change their treatment decision, but the two flexible device constructs were still the most favoured (211/ $566,37.3 \%$ ), followed by a combination of screws and flexible device (hybrid) construct $(114 / 566,20.1 \%)$. Most respondents indicated similar postoperative protocols, regardless of a deltoid injury (6 weeks to weight-bearing, 12 weeks to running, and 16 weeks to full participation; Fig. 2).

\section{Very high impact athlete}

These athletes (e.g. a 300-lb American football offensive or defensive linemen) were consistently treated with a two flexible device construct or a hybrid construct regardless of the presence of a deltoid injury (179/ 566, 31.6\% and 138/566, 24.4\%). Most common preferred times for weight-bearing and running remained the same as the high impact athlete group unless the deltoid was injured. In this case, the postoperative time to full participation increased to 24 weeks (Fig. 3). 

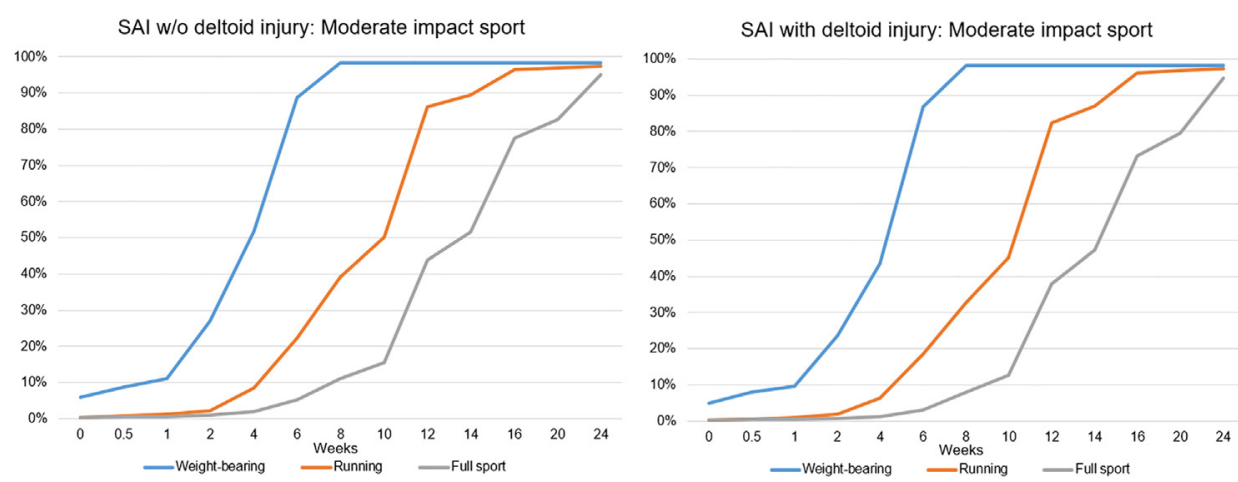

Fig. 1. Cumulative percentage of surgeons' recommended return times to weight-bearing, running, and full sport participation for syndesmotic ankle injuries (SAIs) in moderate impact sports/athletes with vs. without deltoid injury.
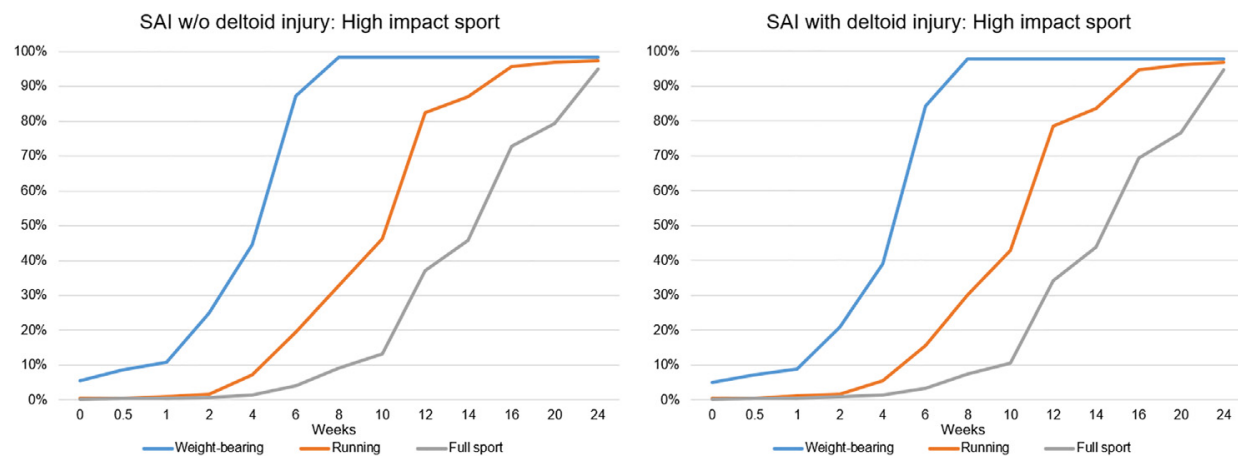

Fig. 2. Cumulative percentage of surgeons' recommended return times to weight-bearing, running, and full sport participation for syndesmotic ankle injuries (SAIs) in high impact sports/athletes with vs. without deltoid injury.
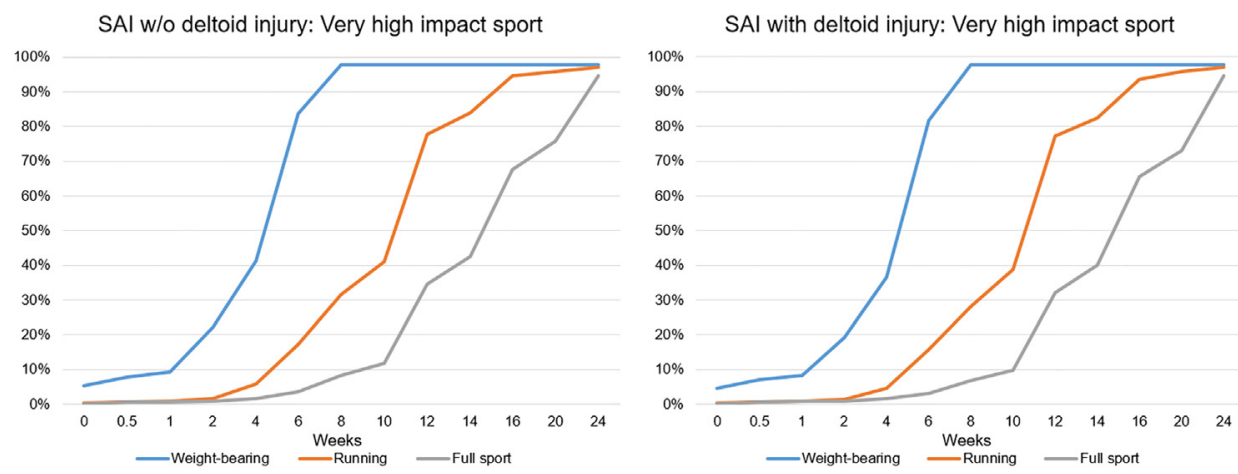

Fig. 3. Cumulative percentage of surgeons' recommended return times to weight-bearing, running, and full sport participation for syndesmotic ankle injuries (SAIs) in very high impact sports/athletes with vs. without deltoid injury.

\section{Common themes}

The use of flexible devices is the most popular method of fixation, irrespective of the athlete scenario, or the presence of a deltoid injury (Fig. 4). In addition, a majority of responding surgeons (340/566, 63.7\%) indicated that they would use the same postoperative protocols for all athlete types and injury patterns presented in this survey (moderate/ high/very high impact athletes with or without a deltoid ligament injury). The results of the survey suggest that although there was consistency among responses at the individual level, there was considerable interrespondent variability with regard to the return to activity times for athletes with syndesmotic injuries.

\section{Discussion}

Overview

The development of standardised protocols for surgical decisionmaking and postoperative rehabilitation of syndesmotic injuries is dependent on proven management strategies for operative indications, techniques, and return to play protocols. The data from this survey indicate both the complexity inherent to surgical indications for these injuries and significant variability in return to activity expectations. The data also indicate a broad preference for flexible fixation devices for these injuries worldwide, consistent with recent outcomes literature [15]. Foremost, 


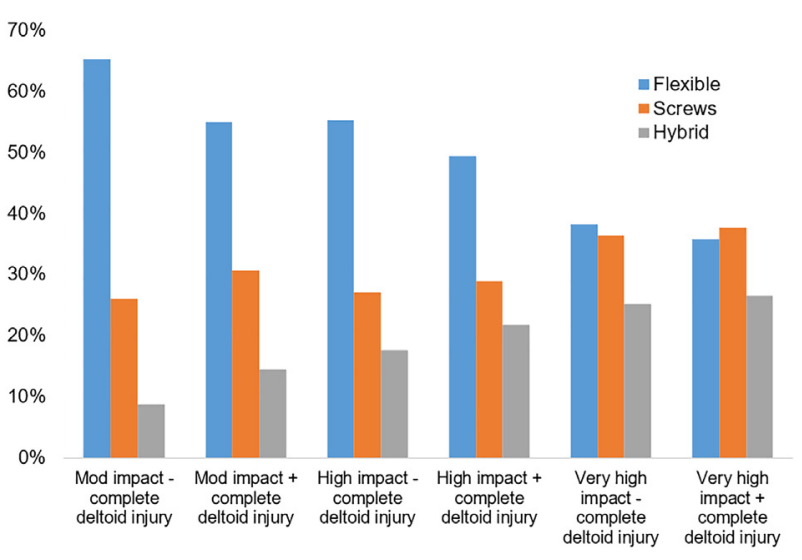

Fig. 4. Orthopaedic surgeons' fixation device preferences during syndesmotic ankle injury (SAI) repair by sport/impact level and deltoid injury status.

these survey data provide a global perspective on how orthopaedic clinicians currently manage syndesmotic injuries in athletes. A prospective evaluation of outcomes, particularly inclusive of those provider teams with a more aggressive return to sport protocol, will add substantial value to safe and expeditious management of syndesmosis injuries.

\section{Indications for operative treatment}

\section{MRI versus radiographs versus arthroscopy}

From our respondent data, it was clear that there is no widely accepted MRI finding as a sole indication for operative repair of the injured syndesmosis. Instead, MRI is used most commonly to examine ligamentous injuries in combination with other examination modalities to determine the best course of treatment. To optimise the use of MRI findings to predict instability and therefore surgical indications, adoption of an MRI classification system, such as the one proposed by Sikka et al. (2012), may be an effective means to formulate a greater consensus on indications for operative management of syndesmotic injuries [16]. The most widely accepted indications were the presence of an AITFL in combination with either a PITFL $(89.7 \%)$ or deltoid ligament $(89.5 \%)$ injury (Table 2). These injury patterns correlate with Sikka Grade III and IV injuries on MRI (Grade III: AITFL + IOM + PITFL; Grade IV: all ligaments + deltoid) [16].

In contrast to MRI findings, the use of radiography to dictate the management of syndesmotic injuries was much more prevalent in our survey results. We found that the two most agreed on radiographic indications for operative fixation of a syndesmotic injury were medial clear space widening $>2 \mathrm{~mm}$ on stress radiography $(437 / 611,71.5 \%)$ and syndesmotic widening $>2 \mathrm{~mm}$ on a weight-bearing radiograph (461/ $617,74.7 \%$; Table 2). Based on the responses from participating surgeons across the globe, we propose that an agreement of $71-75 \%$ on these radiographic metrics warrants general acceptance of these two findings as sole indications for operative treatment.

Arthroscopic evaluation of syndesmotic injuries is a valuable intraoperative tool to examine the stability of the joint and assess the extent of the injury. The use of this technique allows the surgeon to visualise subtle displacement across different planes and more accurately determine whether the injury warrants surgical stabilisation [17]. Survey respondents agreed with the value placed in arthroscopy for determining the extent of a syndesmotic injury, with $68.6 \%(410 / 598)$ agreeing that the discovery of syndesmotic widening was a sole indication for operative repair (Table 2). With greater than two-thirds agreement on the use of syndesmotic widening seen on arthroscopy influencing operative decision-making, we found that clinicians generally accept arthroscopy as a diagnostic tool. Importantly, it is common practice in many regions to use arthroscopic evaluation to confirm instability when it is suspected, rather than use it as a primary diagnostic tool. However, office-based arthroscopic techniques may impact this paradigm [18].

\section{Physical examination}

The clinical examination is vital to correctly diagnosing syndesmotic injuries and rating their severity. The most commonly used diagnostic tests include the squeeze, external rotation, lateral hook, and Cotton tests. Respondent data indicated that $40.1 \%(244 / 609)$ of surgeons used a positive Cotton test as a sole indication for surgery (Table 2). The remaining responses showed variability in what orthopaedic clinicians believed was the most appropriate sole indication for operative fixation. Because there was no agreement in greater than half of the responses gathered, we conclude that physical examination findings are used in conjunction with one another, and no single examination findings or diagnostic test is generally agreed on as having more utility compared with others.

\section{Deltoid injury and foot alignment}

From the survey findings, it can be concluded that most respondents (61.6\%) infrequently (less than half of the time or never/almost never) surgically address complete deltoid injuries. With no clear guidance in the literature, repair of the deltoid ligament remains controversial particularly in the setting of a syndesmotic and deltoid ligament injury without a fracture [19]. A recent systematic literature review by Dabash et al. (2019) determined that there was no consensus on repairing an injured deltoid ligament in the setting of ankle fractures [20]. The clinical outcomes associated with the decision not to repair the deltoid ligament for patients with syndesmotic injuries have yet to be investigated, although biomechanical evidence favours the inclusion of deltoid ligament repair [21,22]. The patient's underlying foot alignment also appeared to have minimal impact on management decisions for clinicians treating syndesmotic injuries. For participants that used foot alignment as a decision-making element, the changes seen in their treatment favoured a conservative postoperative protocol. Differences in postoperative protocol and rehabilitation focused on the use of medial posting orthotics for pes planovalgus feet and orthotics plus peroneal strengthening in pes cavus feet.

\section{Device selection}

\section{Flexible devices versus surgical screws versus hybrid constructs}

Responses fell into one of three categories: screws (one tri-/quadricortical screw, two tri-/quadri-cortical screws, or three or more tricortical screws), flexible devices (one, two, or three or more flexible devices), or hybrid constructs (screws and flexible device combo). Participants also had the option of selecting "None of These." To better understand device preferences as a whole, independent of the type of athlete, we examined all three athlete scenarios together and stratified the data set based on the absence or presence of a complete deltoid injury. In doing so, we found that flexible constructs were highly preferred over screws and hybrid constructs for the moderate and high impact athletes. The traditional method for the fixation of syndesmotic injuries is the use of surgical screws [8]. However, this survey indicates a shift towards the use of flexible devices for the athlete population. Only in the scenario of a very high impact athlete did the choice between which fixation construct was used became variable (Fig. 4). Of note, several of the "none of these" responses indicated preference for a direct anatomic repair of the AITF ligament, with or without a flexible device. Whether this trends grows in popularity remains to be seen.

\section{Return to sport}

Participants surveyed were asked to provide their postoperative weight-bearing, running, and time to full participation protocol for each athlete (moderate, high, or very high impact) under both clinical scenarios (syndesmotic injury alone or syndesmotic and deltoid ligament injury). Among the survey respondents, there was consistency in return to activity times on the individual level, with $63.8 \%$ of responding participants (340/533) keeping the same rehabilitation protocol regardless of injury severity or athlete type. As a result, the respondents who preferred quicker or longer return to sport protocols did so consistently 
across all athletes and injury patterns.

Recent literature suggests that postoperative protocols after fixation of a high-grade syndesmosis injury is 4-6 weeks non-weight-bearing and return to sport at 10-12 weeks based on injury severity [8]. However, we found considerable interrespondent variability in return to activity timelines. Response times across all participants ranged from immediately to 8 weeks for weight-bearing, 2-16 weeks for running, and 6-24 weeks for full participation for the same athlete scenario (Figs. 1-3). In addition, the presence of a deltoid injury resulted in interrespondent variability by prolonging the time to full participation by 4-8 weeks. Therefore, we determined that there was no consensus for the return to activity protocol among respondents.

\section{Limitations}

There are limitations inherent to all survey studies. One limitation to the survey was we did not make all of the survey items a requirement for completion. This explains the differences in the denominator of the proportions of individual items for sections reported throughout the paper. It is possible that a portion of the surveyed participants are members of multiple organisations. As a result, some respondents may have received the survey through different societies, providing them an additional attempt at completing the survey. Because the survey responses were anonymous, it was not possible to delete a second entry by an individual respondent. Another potential limitation is the possibility for selection bias and failure of generalizability. We helped offset these possible limitations by surveying members of various international societies, gathering a global perspective on the management of syndesmotic injuries.

\section{Conclusion}

We examined the indications for operation, device choice, and postoperative management of different types of athletes suffering isolated syndesmotic ligament injury with or without a deltoid ligament involvement. We found consistency regarding the diagnostic criteria indicating surgical intervention. The most commonly used elements were syndesmotic widening $>2 \mathrm{~mm}$ on $\mathrm{x}$-ray, an AITFL injury in combination with a PITFL or deltoid ligament involvement on MRI, and widening of the distal tibiofibular joint during arthroscopic evaluation. The majority of surgeons selected flexible fixation or a hybrid construct of screw and flexible device, regardless of the athlete type or injury pattern. There was considerable variability between respondents on expected postoperative rehabilitation, including time to weight-bearing, running, and full sports participation. Interestingly, the intrarespondent postoperative protocols were consistent across all athletes and injury patterns on the individual respondent level. Prospective study of outcomes after surgical management of syndesmotic injuries will be helpful to determine optimal implant constructs and safe and expeditious return to play protocols.

\section{Funding}

The International Society of Arthroscopy, Knee Surgery and Orthopaedic Sports Medicine provided funding to support this project.

\section{Authors' contributions}

All authors contributed to the writing of the article. D.M., P.P., P.D., A.A., and K.H. contributed to the planning and development of the survey. M.T. is responsible for the statistics.

\section{Declaration of competing interest}

K.J.H. reports other from Panther Orthopaedics, during the conduct of the study; In addition, he has a patent Puma issued. The other authors have no competing interests to declare.

\section{Appendix A. Supplementary data}

Supplementary data to this article can be found online at https ://doi.org/10.1016/j.jisako.2021.10.005.

\section{References}

[1] Waterman BR, Belmont Jr PJ, Cameron KL, Svoboda SJ, Alitz CJ, Owens BD. Risk factors for syndesmotic and medial ankle sprain: role of sex, sport, and level of competition. Am J Sports Med 2011;39(5):992-8. https://doi.org/10.1177/ 0363546510391462.

[2] Hunt KJ, George E, Harris AH, et al. Epidemiology of syndesmosis injuries in intercollegiate football: incidence and risk factors from National Collegiate Athletic Association injury surveillance system data from 2004-2005 to 2008-2009. Clin J Sport Med 2013;23(4):278-82. https://doi.org/10.1097/JSM.0b013e31827ee829.

[3] Sman AD, Hiller CE, Rae K, et al. Diagnostic accuracy of clinical tests for ankle syndesmosis injury. Br J Sports Med 2015;49(5):323-9. https://doi.org/10.1136/ bjsports-2013-092787.

[4] Wolf BR, Amendola A. Syndesmosis injuries in the athlete: when and how to operate. Curr Opin Orthop 2002;13(2):151-4. https://doi.org/10.1097/00001433200204000-00013.

[5] Coetzee JC, Giza E, Schon LC, et al. Treatment of osteochondral lesions of the talus with particulated juvenile cartilage. Foot Ankle Int 2013;34(9):1205-11. https:// doi.org/10.1177/1071100713485739.

[6] Klitzman R, Zhao H, Zhang L-Q, Strohmeyer G, Vora A. Suture-button versus screw fixation of the syndesmosis: a biomechanical analysis. Foot Ankle Int 2010;31(1): 69-75. https://doi.org/10.3113/FAI.2010.0069.

[7] Kortekangas T, Savola O, Flinkkilä $\mathrm{T}$, et al. A prospective randomised study comparing TightRope and syndesmotic screw fixation for accuracy and maintenance of syndesmotic reduction assessed with bilateral computed tomography. Injury 2015;46(6):1119-26. https://doi.org/10.1016/ j.injury.2015.02.004.

[8] Hunt KJ, Phisitkul P, Pirolo J, Amendola A. High ankle sprains and syndesmotic injuries in athletes. JAAOS-Journal of the American Academy of Orthopaedic Surgeons 2015;23(11):661-73. https://doi.org/10.5435/JAAOS-D-13-00135.

[9] Schepers T. Acute distal tibiofibular syndesmosis injury: a systematic review of suture-button versus syndesmotic screw repair. Int Orthop 2012;36(6):1199-206. https://doi.org/10.1007/s00264-012-1500-2.

[10] Williams GN, Jones MH, Amendola A. Syndesmotic ankle sprains in athletes. Am J Sports Med 2007;35(7):1197-207. https://doi.org/10.1177/0363546507302545.

[11] Andersen MR, Frihagen F, Hellund JC, Madsen JE, Figved W. Randomized trial comparing suture button with single syndesmotic screw for syndesmosis injury. JBJS 2018;100(1):2-12. https://doi.org/10.2106/JBJS.16.01011.

[12] DeGroot H, Al-Omari AA, Ghazaly SAE. Outcomes of suture button repair of the distal tibiofibular syndesmosis. Foot Ankle Int 2011;32(3):250-6. https://doi.org/ 10.3113/FAI.2011.0250.

[13] van Vlijmen N, Denk K, van Kampen A, Jaarsma RL. Long-term results after ankle syndesmosis injuries. Orthopedics 2015;38(11):e1001-6. https://doi.org/10.3928/ 01477447-20151020-09.

[14] Mak MF, Gartner L, Pearce CJ. Management of syndesmosis injuries in the elite athlete. Foot Ankle Clin 2013;18(2):195-214. https://doi.org/10.1016/ j.fcl.2013.02.002.

[15] Shimozono Y, Hurley ET, Myerson CL, Murawski CD, Kennedy JG. Suture button versus syndesmotic screw for syndesmosis injuries: a meta-analysis of randomized controlled trials. Am J Sports Med 2019;47(11):2764-71. https://doi.org/10.1177/ 0363546518804804.

[16] Sikka RS, Fetzer GB, Sugarman E, et al. Correlating MRI findings with disability in syndesmotic sprains of NFL players. Foot Ankle Int 2012;33(5):371-8. https:// doi.org/10.3113/FAI.2012.0371.

[17] Watson BC, Lucas DE, Simpson GA, Berlet GC, Hyer CF. Arthroscopic evaluation of syndesmotic instability in a cadaveric model. Foot Ankle Int 2015;36(11):1362-8. https://doi.org/10.1177/1071100715589631.

[18] Stornebrink T, Altink JN, Appelt D, Wijdicks CA, Stufkens SAS, Kerkhoffs G. Twomillimetre diameter operative arthroscopy of the ankle is safe and effective. Knee Surg Sports Traumatol Arthrosc 2020:1-7. https://doi.org/10.1007/s00167-02005889-7.

[19] McCollum GA, van den Bekerom MP, Kerkhoffs GM, Calder JD, van Dijk CN. Syndesmosis and deltoid ligament injuries in the athlete. Knee Surg Sports Traumatol Arthrosc 2013 Jun;21(6):1328-37. https://doi.org/10.1007/s00167012-2205-1.

[20] Dabash S, Elabd A, Potter E, Fernandez I, Gerzina C, Thabet AM, McGarvey W, Abdelgawad A. Adding deltoid ligament repair in ankle fracture treatment: is it necessary? A systematic review. Foot Ankle Surg 2019 Dec;25(6):714-20. https:// doi.org/10.1016/j.fas.2018.11.001.

[21] Butler BA, Hempen EC, Barbosa M, et al. Deltoid ligament repair reduces and stabilizes the talus in unstable ankle fractures. J Orthop 2020;17:87-90. https:// doi.org/10.1016/j.jor.2019.06.005.

[22] Mococain P, Bejarano-Pineda L, Glisson R, et al. Biomechanical effect on joint stability of including deltoid ligament repair in an ankle fracture soft tissue injury model with deltoid and syndesmotic disruption. Foot Ankle Int 2020;41(9): 1158-64. https://doi.org/10.1177/1071100720929007. 